\title{
Proposal for modelling and numerical simulation with SCILAB for learning the continuous and discontinuous dynamics of biological and anaerobic digesters
}

\author{
F.A. Leon ${ }^{\mathrm{a}, *}$, A. Ramos Martinn ${ }^{\mathrm{a}}$, S. Brito Espino ${ }^{\mathrm{b}}$, C. Mendieta Pino ${ }^{\mathrm{a}}$, \\ Tania Garcia-Ramirez ${ }^{\mathrm{a}}$ \\ aDepartamento de Ingeniería de Procesos, Universidad de Las Palmas de Gran Canaria, 35017, \\ Campus Universitario de Tafira, Spain, emails: federico.leon@ulpgc.es (F.A. Leon), \\ alejandro.ramos@ulpgc.es (A. Ramos Martin), carlos.mendieta@ulpgc.es (C. Mendieta Pino), \\ tania.garcia106@alu.ulpgc.es (T. Garcia-Ramirez) \\ ${ }^{b}$ Instituto Universitario de Investigación en Estudios Ambientales y Recursos Naturales, Universidad, \\ de Las Palmas de Gran Canaria, 35017 Campus Universitario de Tafira, Spain, email: saulo.brito09@gmail.com
}

Received 14 October 2020; Accepted 24 June 2021

\begin{abstract}
A B S T R A C T
This article presents a proposal for modelling and numerical simulation with SCILAB with the aim of learning the continuous and discontinuous dynamics of anaerobic biological digesters. The objective is for the proposed design to be used in relevant Bachelor's and Master's degree course topics related to the area of environmental technology. The design is characterised by its versatility of the use in different situations and its employment of free and open-source tools. This latter aspect is an important characteristic as it allows the design to be modified quickly by students or teachers, depending on future needs. Some example simulation results are shown of the dynamic response of both continuous and discontinuous operation according to predefined operation specifications. By demonstrating these simulations, the design can be used to understand and learn the proposed objective.
\end{abstract}

Keywords: Biological kinetics; Simulation; Model; Numerical method

\section{Introduction}

Anaerobic digestion has been established as a satisfactory process for the stabilisation of effluent with a high organic load and it is important to know the different factors that affect the design of the biological reactor. Anaerobic treatment systems are biological systems that operate in the absence of oxygen and are very suitable for the treatment of highly biodegradable waste. Therefore, it is of interest to design learning strategies, based on information and communication technologies (ICTs), to help students in relevant Bachelor's and Master' degree courses and

* Corresponding author. provide them with skills in environmental technologies to learn and understand the evolution of the relevant variables in the operation of anaerobic digesters, both continuous and discontinuous. Such learning strategies can be based on the use of experimental and laboratory artefacts or designs, as well as virtual implementations by means of simulations, under easily modifiable environments, designed for the study and analysis of the issues of concern. The educational proposal related to artefacts is based on psychological learning theories developed in different works [1-4], while the second proposal is based on simulations and is set out in the following references [5-9]. In this way, it is 
possible to achieve appropriate experimental environments in line with a constructivist approach to learning through active techniques $[1,10]$.

The main objective of this article is a proposal of modelling and numerical simulation with SCILAB, with a view to learning the continuous and discontinuous dynamics of anaerobic biological digesters.

\section{Fundamentals of biological process modelling}

Bacteria, and to a lesser extent fungi, are responsible for the biodegradation reactions that take place in biotreatment [11-14]. Although most degradation reactions are part of the normal metabolism of these cells, the goal of microorganism metabolism is not to remove environmental contaminants, but to grow and sustain the microorganisms. Therefore, the formulation of models must begin with the active biomass and the factors that allow this growth and maintenance.

A biological wastewater treatment or purification process is a system in which a culture of micro-organisms (biomass) must be maintained, feeding on the impurities in the wastewater (substrate or food). The microorganisms grow and feed by extracting nutrients, electrons, and energy from the environment. These impurities are the biodegradable organic matter (ammonium, nitrate, phosphate, and other contaminants at lower concentrations) and form the basis of the cellular constituents (carbohydrates, amino acids, lipids, and nucleic acids). Electrons are necessary to reduce the nutrients to the chemical form used by the cellular constituents and to generate the energy necessary to make the synthesis and the maintenance of the biomass possible. The most basic process of microbial metabolism is the transfer of electrons from a donor substrate to a receptor substrate.

The place where the biomass is brought into contact with the wastewater to carry out the treatment is called a biological reactor or digester, also known as a bioreactor or biodigester, and can be of different types.

\subsection{Basic components of the model}

In the simplest mathematical model, at least two components are required to describe what happens in a digester. These components are:

- Substrate $(S)$ : component of the wastewater that serves as food for the biomass. In a heterotrophic process, its concentration can be measured through the biochemical oxygen demand $\left(\mathrm{BOD}_{5}\right)$ or the chemical oxygen demand (COD). In fact, this model considers that only soluble organic matter is biodegradable and can be attacked by biomass, and so $S$ is a soluble component. The substrate, therefore, is not separated by sedimentation.

- Biomass $(X)$ : culture of microorganisms that feed on the biodegradable organic matter in the wastewater. Its concentration can be measured by the concentration of volatile suspended solids (VSS) in the biological digester. The biomass is formed by particles and can be separated by sedimentation, filtration or flotation.

\subsection{Basic kinetic processes}

A simple two-component model, as described above, has at least two basic processes:

- Biomass growth: this process is responsible for the consumption of the substrate and the growth of the biomass.

- Death of the biomass: this process implies a reduction in the growth of the biomass obtained in the previous process.

\subsection{Basic model of a microbiological system}

Let us suppose that the growth, at the expense of a single substrate $S$, of the biomass of microorganisms $X$ in a perfectly mixed digester is represented by the reaction:

$S \rightarrow X$

where $S$ is the limiting substrate for microbial growth and $X$ is the active biomass. The biomass growth utilization rate can be expressed as:

$\frac{d X}{d t}=\mu \cdot X$

This is a first-rate process, while the speed of consumption of the substrate will be given by:

$\frac{d S}{d t}=-k \cdot S$

where $k$ is the specific rate of substrate consumption $(S-1)$ and $\mu$ is the specific rate of microbial growth $(S-1)$. With this we obtain a system of differential equations:

$$
\left\{\begin{array}{l}
\frac{d x}{d t}=\mu \cdot X \\
\frac{d S}{d t}=-k \cdot S
\end{array}\right.
$$

This system constitutes the mathematical representation of the system under consideration. If we express both processes (growth of biomass and consumption of substrate) as a function of the speed of growth of the biomass, Eq. (4) would be transformed into:

$$
\left\{\begin{array}{l}
\frac{d x}{d t}=\mu \cdot X \\
\frac{d S}{d t}=-\alpha \cdot \mu \cdot S \cdot X
\end{array}\right.
$$

where $\alpha$ is a stoichiometric coefficient for the state variable $S$. The stoichiometric coefficients provide the value of the conversion factor between the process speed and the speed of variation, induced by it, in each state variable.

It is important to highlight that the biomass of microorganisms plays the role of self-catalyst, since it is produced in the process, but its presence is necessary for the biotransformation of the substrate to take place. 
Experiments on the growth of microorganisms show that the speed of growth varies with time and is influenced by many physical-chemical and biological environmental factors, including the concentration of substrates $(S)$, concentration of biomass $(X)$, concentration of products $(P), \mathrm{pH}$, temperature $(T)$, concentration of dissolved oxygen, light intensity, and various inhibitors of microbial growth [15].

The specific growth rate of microorganisms is generally expressed as products of individual terms, each of which refers to a growth-limiting factor:

$$
\begin{aligned}
\mu(S, X, P, \mathrm{pH}, T)= & \mu_{\max } \cdot f_{s}(S) \cdot f_{x}(X) \cdot f_{P}(P) . \\
& f_{\mathrm{O}_{2}}\left(\mathrm{O}_{2}\right) \cdot f_{\mathrm{pH}}(\mathrm{pH}) \cdot f_{T}(T)
\end{aligned}
$$

where $\mu_{\max }$ is the maximum specific speed of microbial growth $\left(\mathrm{s}^{-1}\right)$.

As for the type of kinetics used, it is quite common to use Monod's model (7) for enzyme-catalysed processes, so:

$\mu=\mu_{\max } \cdot \frac{S}{K_{s}+S}$

where $K_{s}$ is the semi-saturation constant for the substrate $S$. According to this model, the speed of a biological process of microbial growth will asymptotically tend to the maximum value $\mu_{\max ^{\prime}}$ and the concentration at which the processing speed is equal to half the maximum speed will be the value of the semi-saturation constant of the process $K_{s}$.

It should be clarified that all the biological processes presented in this article are carried out inside a digester, so that the kinetic equations given can be used to obtain the basic mathematical model. It is assumed that the digester is perfectly mixed; that the speed of microbial growth is only affected by the concentration of the available substrate; that it operates in a process of reducing the active biomass, with the speed $\rho_{d}$ directly proportional to the value of the latter:

$\rho_{d}=-k_{d} \cdot X$

that the system (digester) is fed with a current with a substrate concentration $S_{\text {in }}$ of dilution ratio $D$ (the dilution ratio represents the volumetric flow rate of the influent per unit volume of the digester and is expressed as $D=Q / V$, where $V$ is the volume of the digester $\left(\mathrm{m}^{3}\right)$ and $Q$ is the volumetric flow rate of the tributary $\left.\left(\mathrm{m}^{3} \mathrm{~s}^{-1}\right)\right)$ and; that a current of dilution ratio $D$, with the concentration of substrate $S$ and concentration of microorganisms $X$, comes out of the digester.

The application in the digester of material balances for the substrate and the concentration of biomass provides us with the mathematical model that represents the evolution in time of these two components in the digester and comprises the following system of differential equations:

$$
\left\{\begin{array}{l}
\frac{d X}{d t}=\left(\mu-D-k_{d}\right) \cdot X=\left(\mu_{\max } \cdot \frac{S}{K_{s}+S}-D-k_{d}\right) \cdot X \\
\frac{d S}{d t}=D \cdot\left(S_{\text {in }}-S\right)-\beta_{s} \cdot \mu \cdot X=D \cdot\left(S_{\text {in }}-S\right)-\beta_{s} \cdot \mu_{\max } \cdot \frac{S}{K_{s}+S} \cdot X
\end{array}\right.
$$

with this being the most basic model that can be dealt with.

\subsection{Stationary regime of the basic model}

To obtain the steady-state of the system, the system of differential Eqs. (9) must be considered and the derivatives forced to zero, which implies that the steady-state has been reached.

$$
\left\{\begin{aligned}
0 & =\left(\mu_{\mathrm{max}} \cdot \frac{S_{\mathrm{ss}}}{K_{s}+S_{\mathrm{ss}}}-D-k_{d}\right) \cdot X_{\mathrm{ss}} \rightarrow S_{\mathrm{ss}} \\
& =\left(D+k_{d}\right) \cdot \frac{K_{s}}{\mu_{\max }-\left(D+k_{d}\right)} \rightarrow S_{\mathrm{ss}}=f(D) \\
0 & =D \cdot\left(S_{\mathrm{in}}-S_{\mathrm{ss}}\right)-\beta_{s} \cdot \mu_{\mathrm{max}} \cdot \frac{S_{\mathrm{ss}}}{K_{s}+S_{\mathrm{ss}}} \cdot X_{\mathrm{ss}} \rightarrow X_{\mathrm{ss}} \\
& =D \cdot \frac{S_{\mathrm{in}}-S_{\mathrm{ss}}}{\mu \cdot \frac{S_{\mathrm{re}}}{K_{s}+S_{\mathrm{ss}}}} \rightarrow X_{\mathrm{ss}}=f\left(D, S_{\mathrm{in}}\right)
\end{aligned}\right.
$$

As can be seen in Eq. (10), the steady-state depends on the biological properties $\left(k_{d^{\prime}} K_{s^{\prime}} \mu_{\text {max }}\right)$, the $D$ dilution, and the input concentration of the substrate $S_{\text {in }}$. In addition, the following conditions must be met to obtain a real solution:

$\mu_{\max }>k_{d}$

$D<\mu_{\max }-k_{d}$

These conditions are necessary because if the maximum growth rate $\mu_{\max }$ is not higher than the biomass decline there will be no stable population and no anaerobic digestion. Something similar occurs with the dilution, which must be lower than the concentration of the active biological population. Therefore, the maximum ideal dilution will be $D=\mu_{\max }-k_{d}$.

\section{Basic model with impulse type input}

The operation of the digester with a continuous input is as described above, but the discontinuous loading of substrate requires a series of modifications to the model [16-19] to be taken into account. This section will show the approach of the model for a type of loading that is in effect an impulse input, since loading implies the introduction in a very short time period of a specific volume, $V_{1}$, with an organic load. This loading will be repeated each interval of time $T$, which is much greater than a load time that effectively tends to zero with respect to $T$. The volume $V_{1}$ of the load, each time it is introduced, displaces an amount of volume equal to $V_{1}$, with the total volume of the digester being $V_{T}$ and the non-displaced volume $V_{2^{\prime}}$ such that $V_{T}=V_{1}+V_{2}$. The volume of liquid with the organic matter is assumed to be perfectly mixed at the same instant as the loading is concluded. Because of the above, the dilution will now be expressed as follows:

$D=\frac{\hat{Q}}{V_{1}+V_{2}}=\frac{\frac{V_{1}}{T}}{V_{1}+V_{2}}=\frac{\frac{V_{1}}{T}}{V_{T}}$ 
where it can be seen that the flow rate $Q$ is, in fact, a "pseudo flow", as it is a kind of average flow rate in the period $T$, between load and substrate load.

From all the above in relation to the impulse type input, the basic model of Eq. (9) can be adapted to Eq. (16):

$$
\begin{gathered}
\left\{\begin{array}{c}
\frac{d X}{d t}=\left(\mu-k_{d}\right) \cdot X \\
\frac{d S}{d t}=-\beta_{s} \cdot \mu X
\end{array}\right\} \rightarrow t \neq n \cdot T \\
\left\{\begin{array}{c}
\Delta S=\frac{V_{1}}{V_{T}} \cdot\left(S_{\text {in }}-S^{*}(n \cdot T)\right) \\
\Delta X=0
\end{array}\right\} \rightarrow t=n \cdot T
\end{gathered}
$$

where $S^{*}(n \cdot T)$ is the concentration of the substrate just before loading. The concentration that remains after loading and mixing, $S(n \cdot T)$, is expressed as follows:

$S(n \cdot T)=\frac{S_{\text {in }} \cdot V_{1}+S^{*}(n \cdot T) \cdot V_{2}}{V_{T}}$

Eq. (15) is a function of the input concentration, of the concentration in the digester, and their respective volumes.

\section{Scope of the modeling learning proposal}

In this paper, a modelling and solving numerical framework for anaerobic digestion with continuous and discontinuous dynamics is introduced which allows the coupling of different types of processes: biological, physico-chemical, inhibitions, several oxidation and reduction reactions, and various aspects of advanced oxidation $[1,20]$. In addition, multiple reaction conditions, which affect the degradation rate, can be simultaneously considered $[1,20]$. However, while such problems far exceed the learning objectives in the proposed framework of this paper, the intention is to address them in future works. One problem of considerable interest is that of biogas generation. Tackling this question would mean having to increase the number of equations of the system presented in this article, as shown in [21], as this type of problem has a higher complexity in its numerical resolution than that in the systems of the differential Eqs. (9) and (14). Given that the main objective of this work is to show and obtain a simple method to learn the continuous and discontinuous dynamics of anaerobic digestion (while addressing the understanding of the mathematical model and its numerical resolution, which will be discussed in the next section), it is convenient to use the minimum set of relations or equations, both from the point of view of its dynamics (continuous or discontinuous) and from the point of view of numerical resolution. In this way, students will be better able to learn the basic skillsets required, which will enable them in the future to tackle more complex problems independently.

\section{Numerical approach to the basic model}

Since the systems of Eqs. (9) and (14) show non-linear relationships in the state variables, it is necessary to apply numerical approximations to solve them. This implies the application of the typical procedures of initial value problems. In this article, it is proposed to use the predictor-corrector method, which is based on the use of an explicit solution as an initial predictive solution, and then from this, an implicit solution will be used iteratively until the convergence of the solution is achieved with a certain tolerance. The solution used for the predictor is the simplest form of the Euler equation, in its explicit form:

$$
\begin{aligned}
\frac{d y(t)}{d t} & =f(t, y(t)), y\left(t_{0}\right)=y_{0} \rightarrow y_{n+1}=y_{n}+h \cdot f\left(t_{n}, y_{n}\right), \\
t_{n} & =t_{0}+n \cdot h
\end{aligned}
$$

For the corrector, the Crank-Nicolson implicit solution is used, also equivalent to a trapezoidal solution:

$$
\begin{aligned}
& \frac{d y(t)}{d t}=f(t, y(t)), y\left(t_{0}\right)=y_{0} \rightarrow y_{n+1}= \\
& y_{n}+\frac{h}{2} \cdot\left[f\left(t_{n}, y_{n}\right)+f\left(t_{n+1}, y_{n+1}\right)\right], t_{n}=t_{0}+n \cdot h
\end{aligned}
$$

Unifying the two solutions in the prediction-correction process, we have:

$$
\begin{gathered}
y_{n+1}^{(P)}=y_{n}+h \cdot f\left(t_{n}, y_{n}\right) \leftarrow \text { Prediction step } \\
y_{n+1}^{\left(C_{0}\right.}=y_{n}+\frac{h}{2} \cdot\left[f\left(t_{n}, y_{n}\right)+f\left(t_{n+1}, y_{n+1}^{(P)}\right)\right] \leftarrow \text { Initialization step } \\
\text { with the predictor } y_{n+1}^{(P)} \\
y_{n+1}^{\left(C_{k+1}\right.}=y_{n}+\frac{h}{2} \cdot\left[f\left(t_{n}, y_{n}\right)+f\left(t_{n+1}, y_{n+1}^{\left(C_{k}\right)}\right)\right] \leftarrow \text { Recursive correction } \\
\text { step while }\left|y_{n+1}^{\left(C_{k+1}\right.}-y_{n+1}^{\left(C_{k} \mid\right.}\right|>\epsilon
\end{gathered}
$$

As indicated, this iterative process will continue until the convergence specifications are met. Below, this process is presented for the Eq. (14) system.

For the proposed system of equations it would be as follows, with $h=\Delta t$ :

- For the biomass $X$ :

$$
\begin{aligned}
& \frac{X_{n+1}-X_{n}}{\Delta t}=\left(\mu_{\max } \cdot \frac{S_{n}}{K_{s}+S_{n}}-k_{d}\right) \cdot X_{n} \rightarrow \text { Explicit } \\
& \frac{X_{n+1}-X_{n}}{\Delta t}=\left(\mu_{\max } \cdot \frac{S_{n+1}}{K_{s}+S_{n+1}}-k_{d}\right) \cdot X_{n+1} \rightarrow \text { Implicit } \\
& \frac{X_{n+1}-X_{n}}{\Delta t}=\frac{1}{2} \cdot\left[\begin{array}{l}
\left(\mu_{\max } \cdot \frac{S_{n}}{K_{s}+S_{n}}-k_{d}\right) \cdot X_{n}+ \\
\left(\mu_{\max } \cdot \frac{S_{n+1}}{K_{s}+S_{n+1}}-k_{d}\right) \cdot X_{n+1}
\end{array}\right] \rightarrow \text { Crank-Nicolson }
\end{aligned}
$$


- For the substrate $S$ :

$\frac{S_{n+1}-S_{n}}{\Delta t}=-\beta_{s} \cdot \mu_{\max } \cdot \frac{S_{n}}{K_{s}+S_{n}} \cdot X_{n} \rightarrow$ Explicit

$\frac{S_{n}+1-S_{n}}{\Delta t}=-\beta_{s} \cdot \mu_{\max } \cdot \frac{S_{n+1}}{K_{s}+S_{n+1}} \cdot X_{n+1} \rightarrow$ Implicit

$\frac{X_{n+1}-X_{n}}{\Delta t}=\frac{\beta_{s} \cdot \mu_{\max }}{2} \cdot\left[\begin{array}{l}\frac{S_{n}}{K_{s}+S_{n}} \cdot X_{n}+ \\ \frac{S_{n+1}}{K_{s}+S_{n+1}} \cdot X_{n+1}\end{array}\right] \rightarrow$ Crank-Nicolson

- Applying the predictor-corrector method, we have:

$\square$ Prediction step:

$X_{n+1}^{(P}=\left[1+\Delta t\left(\mu_{\max } \cdot \frac{S_{n}}{K_{s}+S_{n}}-k_{d}\right)\right] \cdot X_{n}$

$S_{n+1}^{(P}=\left[1-\beta_{s} \cdot \mu_{\max } \cdot \frac{X_{n}}{K_{s}+S_{n}} \cdot \Delta t\right] \cdot S_{n}$

$\square \quad$ Initialization step with the predictor vector $\left(S_{n+1}^{(P}, X_{n+1}^{(P}\right)$ :

$X_{n+1}^{\left(C_{0}\right.}=X_{n}+\frac{\Delta t}{2} \cdot\left[\begin{array}{l}\left(\mu_{\max } \cdot \frac{S_{n}}{K_{s}+S_{n}}-k_{d}\right) \cdot X_{n}+ \\ \left(\mu_{\max } \cdot \frac{S_{n+1}^{(P)}}{K_{s}+S_{n+1}^{(P}}-k_{d}\right) \cdot X_{n+1}^{(P}\end{array}\right]$

$S_{n+1}^{\left(C_{0}\right.}=S_{n}-\beta_{s} \cdot \mu_{\max } \cdot \frac{\Delta t}{2} \cdot\left[\frac{S_{n}}{K_{s}+S_{n}} \cdot X_{n}+\frac{S_{n+1}^{(P)}}{K_{s}+S_{n+1}^{(P)}} \cdot X_{n+1}^{(P)}\right]$

Recursive correction step while

$\left[\left|S_{n+1}^{\left(C_{k+1}\right.}-S_{n+1}^{\left(C_{k}\right.}\right|+\left|X_{n+1}^{\left(C_{k+1}\right.}-X_{n+1}^{\left(C_{k}\right.}\right|\right]>\epsilon:$

$X_{n+1}^{\left(C_{k+1}\right.}=X_{n}+\frac{\Delta t}{2} \cdot\left[\begin{array}{l}\left(\mu_{\max } \cdot \frac{S_{n}}{K_{s}+S_{n}}-k_{d}\right) \cdot X_{n}+ \\ \left(\mu_{\max } \cdot \frac{S_{n+1}^{\left(C_{k}\right.}}{K_{s}+S_{n+1}^{\left(C_{k}\right.}}-k_{d}\right) \cdot X_{n+1}^{\left(C_{k}\right.}\end{array}\right]$

$S_{n+1}^{\left(C_{k+1}\right.}=S_{n}-\beta_{s} \cdot \mu_{\max } \cdot \frac{\Delta t}{2} \cdot\left[\frac{S_{n}}{K_{s}+S_{n}} \cdot X_{n}+\frac{S_{n+1}^{\left(C_{k}\right.}}{K_{s}+S_{n+1}^{\left(C_{k}\right.}} \cdot X_{n+1}^{\left(C_{k}\right.}\right]$

The Scilab code for this process is included in the Annex.

\section{Results}

This section shows the results obtained from the modelling and simulation proposal with Scilab. Three simulations were proposed with the same dilution $D=0.05 \mathrm{~s}^{-1}$, an input organic load concentration of $S_{\text {in }}=130 \mathrm{~g} / \mathrm{m}^{3}$, and a total volume of $V_{T}=4 \mathrm{~m}^{3}$.

Fig. 1 shows the first simulation (with continuous dynamics) of the evolution of substrate and biomass, starting from an initial state and, after the transitory process, reaching a stationary state, with the values $S_{\mathrm{re}}=61.6 \mathrm{~g} / \mathrm{m}^{3}$ and $X_{\text {re }}=69.7 \mathrm{~g} / \mathrm{m}^{3}$.

Fig. 2 shows the second simulation (with discontinuous dynamics and period $T=0.5 \mathrm{~h}$ ) of the evolution of substrate and biomass, starting from an initial state and, after the transitory process, reaching a stationary state, with the values $S_{\mathrm{re}}=61.6 \mathrm{~g} / \mathrm{m}^{3}$ and $X_{\mathrm{re}}=69.7 \mathrm{~g} / \mathrm{m}^{3}$.

Fig. 3 shows the third simulation (with discontinuous dynamics and period $T=2.5 \mathrm{~h}$ ) of the evolution of substrate and biomass, starting from an initial state and, after the transitory process, reaching a stationary state, with the values $S_{\mathrm{re}}=61.6 \mathrm{~g} / \mathrm{m}^{3}$ and $X_{\mathrm{re}}=69.7 \mathrm{~g} / \mathrm{m}^{3}$.
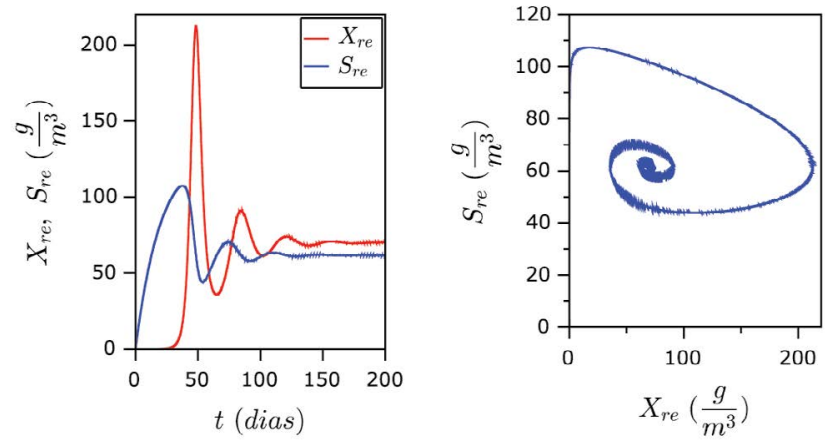

Fig. 1. Result with $S_{\text {re }}=61.6 \mathrm{~g} / \mathrm{m}^{3}$ and $X_{\mathrm{re}}=69.7 \mathrm{~g} / \mathrm{m}^{3}$ and continuous regime.
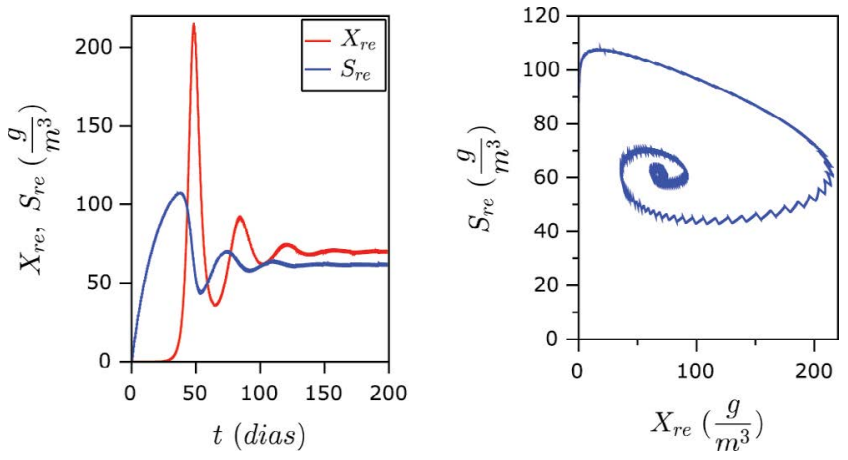

Fig. 2. Result with $S_{\mathrm{re}}=61.6 \mathrm{~g} / \mathrm{m}^{3}$ and $X_{\mathrm{re}}=69.7 \mathrm{~g} / \mathrm{m}^{3}$ and discontinuous regime with $T=0.5 \mathrm{~h}$.
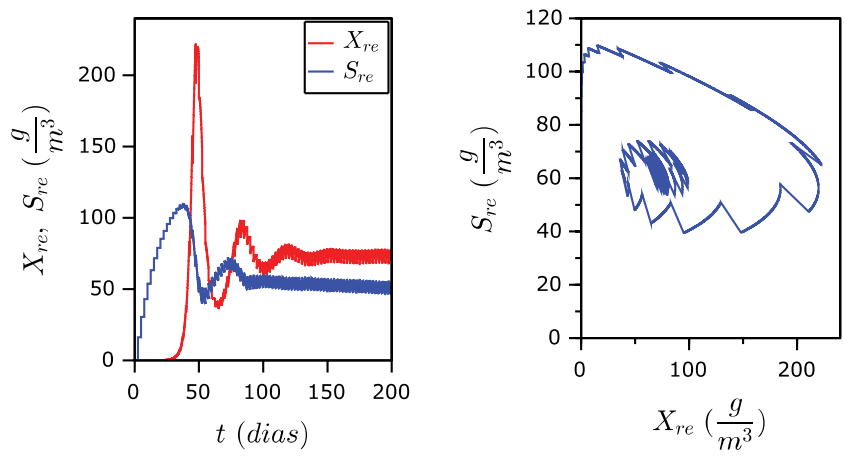

Fig. 3. Result with $S_{\mathrm{re}}=61.6 \mathrm{~g} / \mathrm{m}^{3}$ and $X_{\mathrm{re}}=69.7 \mathrm{~g} / \mathrm{m}^{3}$ and discontinuous regime with $T=2.5 \mathrm{~h}$. 


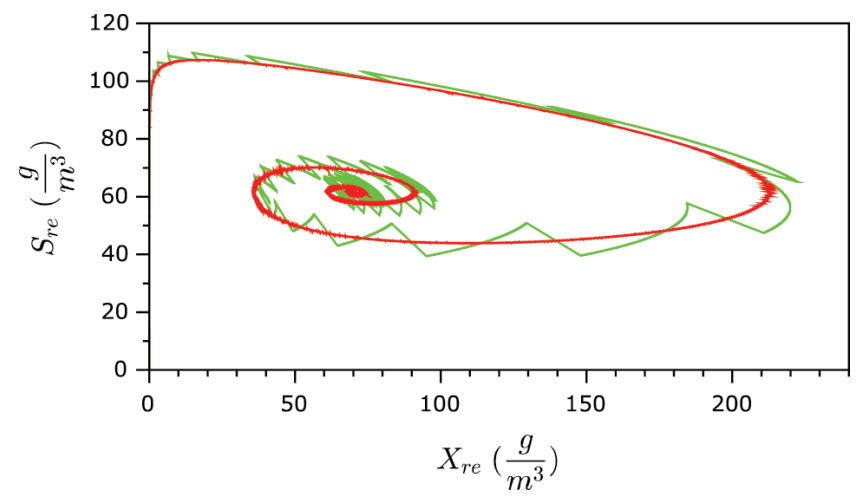

Fig. 4. Results of the first and third simulations.

The first and third simulations are shown together in Fig. 4 for comparison purposes. It can be seen that in both cases the steady-state coincides, as expected.

\section{Conclusions}

This article presents a proposal for modelling and numerical simulation, with the aim of learning the continuous and discontinuous dynamics of anaerobic biological digesters. The design is based on the use of Scilab, an open-source software application. This characteristic makes it possible for studies to easily modify the original design presented in this study. A series of trials were implemented to test the technical and pedagogical feasibility of the proposal. From the experimental results obtained and the tests carried out, it was found that the learning proposal presented in this article is viable. Finally, the proposed experimental design is shown to be extremely flexible and versatile, principally thanks to the use of free applications, its ease of use, and the large community working on the development of new calculation tools within the scope of Scilab.

\section{Acknowledgements}

This research has been co-funded by the INTERREG V-A Cooperation Spain-Portugal MAC (Madeira-AzoresCanarias) programme MITIMAC project MAC2/1.1a/263.

\section{ANNEX. SCILAB CODE.}

clear; clc;

$\operatorname{mu} \max =.1 / 3600$;

$\mathrm{k} \_\mathrm{s}=100 ; \mathrm{k} \_\mathrm{d}=.00001 ; \mathrm{k} \_\mathrm{x}=.00000150$;

$\mathrm{V} \_1=.01 ; \mathrm{V} \_2=3.99 ; \mathrm{V} \_\mathrm{T}=\mathrm{V} \_1+\mathrm{V} \_2$;

S_min $=$ k_d d $^{*}$ _s $/($ mu_max-k_d $) ;$ S_in $=130$;

$\mathrm{dt}=100 ;$ time $=200^{*} 24^{*} 3600 ;$ Delta_t $=.05^{*} 24^{*} 3600$;

$\mathrm{q}=\operatorname{round}($ Delta_t $/ \mathrm{dt})$;

$\mathrm{t}=0$ :dt:time;

$X=$ zeros(1,length $(t)) ; S=$ zeros $(1$,length $(t))$;

$X(1)=.1 ; S(1)=0 ; S \_$in $=S \_$in; $X \_$in $=.1$;

$\mathrm{e}=1 ; \mathrm{f}=0 ; \mathrm{g}=0$;

for $\mathrm{k}=2$ :length $(\mathrm{t})$

$\mathrm{f}=\mathrm{f}+1$;

if $\mathrm{f}>=\mathrm{q} / /$ Impulse input

$$
\mathrm{S}(\mathrm{k}-1)=\left(\mathrm{V} \_2 * \mathrm{~S}(\mathrm{k}-1)+\mathrm{V}_{-} 1^{*} \mathrm{~S} \_ \text {in }\right) / \mathrm{V}_{-} \mathrm{T} \text {; }
$$

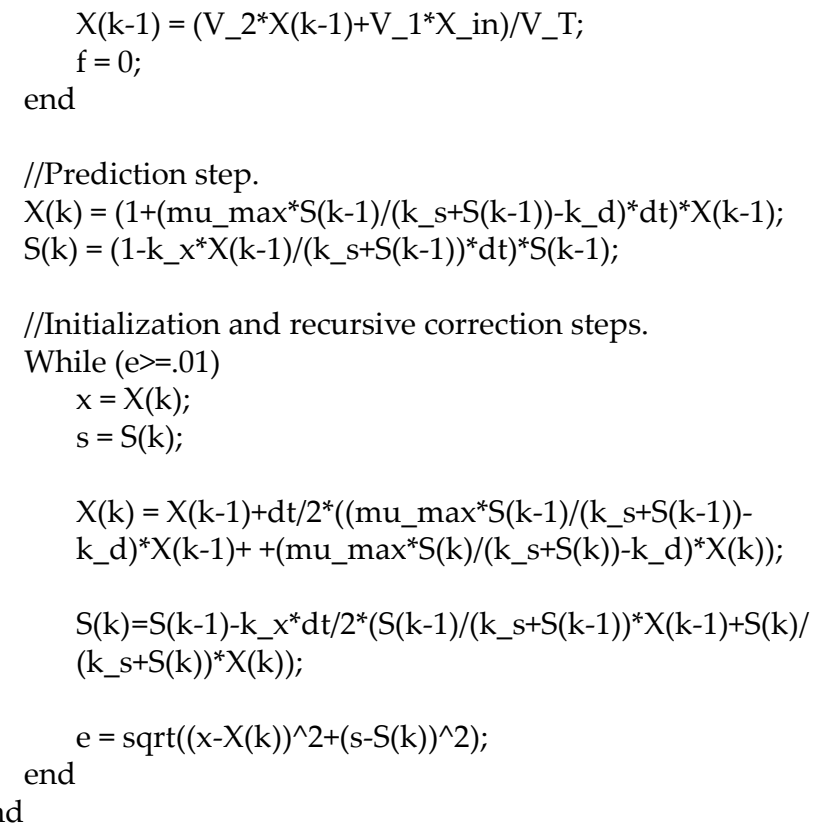

\section{References}

[1] S. Brito-Espino, C.A. Mendieta-Pino, S.O. Pérez-Báez, A. Ramos-Martín, Application of a model-based method for hydrodynamic processes in constructed wetland to management of livestock wastewater based on finite elements method, Desal. Water Treat., 152 (2019) 92-98.

[2] I. Garcia, E.M. Cano, Designing and implementing a constructionist approach for improving the teaching-learning process in the embedded systems and wireless communications areas, Comput. Appl. Eng. Educ., 22 (2014) 481-493.

[3] E.M. Cano, J.G. Ruiz, I.A. Garcia, Integrating a learning constructionist environment and the instructional design approach into the definition of a basic course for embedded systems design, Comput. Appl. Eng. Educ., 23 (2015) 36-53.

[4] K. Sorathia, R. Servidio, Learning and experience: teaching tangible interaction and edutainment, Procedia Soc. Behav. Sci., 64 (2012) 265-274.

[5] D. Ifenthaler, F. Landriscina, Simulation and Learning. A Model-Centered Approach, Springer, Switzerland, 2013.

[6] A.A. Deshpande, S.H. Huang, Simulation games in engineering education: a state-of-the-art review, Comput. Appl. Eng. Educ., 19 (2011) 399-410.

[7] M. Uribe, A.J. Magana, J.H. Bahk, A. Shakouri, Computational simulations as virtual laboratories for online engineering education: a case study in the field of thermoelectricity, Comput. Appl. Eng. Educ., 24 (2016) 428-442.

[8] W.P. Lee, C.L. Hwan, A computer simulation in mechanics teaching and learning: a case study in circular motions, Comput. Appl. Eng. Educ., 23 (2015) 865-871.

[9] A.K. Datta, V. Rakesh, D.G. Way, Simulation as an integrator in an undergraduate biological engineering curriculum, Comput. Appl. Eng. Educ., 21 (2013) 717-727.

[10] R.C. Smith, O.S. Iversen, M. Hjorth, Design thinking for digital fabrication in education, Int. J. Child Comput. Interact., 5 (2015) 20-28.

[11] S. Corbellini, A. Vallan, Arduino-Based Portable System for Bioelectrical Impedance Measurement, 2014 IEEE International Symposium on Medical Measurements and Applications (MeMeA), IEEE, Lisboa, Portugal, 2014, pp. 1-5.

[12] P.A. Pawar, Heart Rate Monitoring System Using IR Base Sensor \& Arduino Uno, 2014 Conference on IT in Business, Industry and Government (CSIBIG), IEEE, Indore, India, 2014, pp. 1-3.

[13] M. Gandra, R. Seabra, F.P. Lima, A low-cost, versatile data logging system for ecological applications, Limnol. Oceanogr. Methods, 13 (2015) 115-126. 
[14] M.A. Gealt, A. Morris, Biotratamiento de residuos t'oxicos y peligrosos, McGraw-Hill Interamericana, New York, USA, 2012.

[15] F.L. Colomer, Metabolismo bacteriano y modelizaci'on matem'atica de procesos, III Jornadas de Innovación Educativa, Las Palmas de Gran Canaria, 2016.

[16] C. Wei, L. Chen, Dynamic analysis of mathematical model of ethanol fermentation with gas stripping, Nonlinear Dyn., 57 (2009) 13-23.

[17] E. Mekic, I. Djokic, S. Zejnelagic, A. Matovic, Constructive approach in teaching of VoIP in line with good laboratory and manufacturing practice, Comput. Appl. Eng. Educ., 24 (2016) $277-287$.

[18] F.A. Leon, A. Ramos. Analysis of high efficiency membrane pilot testing for membrane design optimisation, Desal. Water Treat., 73 (2017) 208-214.
[19] S. Boerlage, N. Nada. Algal toxin removal in seawater desalination processes, Desal. Water Treat., 55 (2015) 2575-2593.

[20] D.J. Bastone, J. Keller, I. Angelidaki, S.V. Kalyuzhnyi, S.G. Pavlostathis, A. Rozzi, W.T.M. Sanders, H. Siegrist, V.A. Vavilin, Anaerobic Digestion Model no 1, IWA Publishing, London, 2002.

[21] A. Wu, D. Lovett, M. McEwan, F. Cecelja, T. Chen, A spreadsheet calculator for estimating biogas production and economic measures for UK-based farm-fed anaerobic digesters, Bioresour. Technol., 220 (2016) 479-489. 